\title{
Adaptações do Cérebro Durante uma Tarefa de Longa Duração: Um Estudo de Potencial Relacionado a Evento.
}

\author{
Hiago Murilo de Melo* (D, Lucas Martins Nascimento, \& Emílio Takase \\ Universidade Federal de Santa Catarina, Florianópolis, SC, Brasil
}

\begin{abstract}
RESUMO - O objetivo deste estudo é investigar o efeito da demanda cognitiva prolongada na modulação do Potencial Relacionado a Evento (ERP) em um paradigma de controle inibitório. Os dados foram coletados em 19 voluntários destros, com a média de idade de $21,21( \pm 1,77)$ anos, que realizaram o paradigma do Go/NoGo durante 50 minutos, com gravação sincronizada do eletroencefalograma para obtenção dos ERPs. O efeito do tempo de realização da tarefa provocou alterações significativas nas variáveis subjetivas, de desempenho cognitivo e nas amplitudes máximas dos componentes N2 e P3. Nossos resultados sugerem que quando nosso cérebro está submetido a demandas cognitivas extensas, ocorrem adaptações para a manutenção do desempenho comportamental através da estratégia de realocação de recursos energéticos.
\end{abstract}

PALAVRAS-CHAVE: tarefa de longa duração, controle inibitório, ERPs, N2, P3

\section{Brain Adaptations During a Long-time Task: An Event-Related Potential (ERP) Study.}

\begin{abstract}
The aim of this study is to investigate the effect of prolonged cognitive demand in Event Related Potential (ERP) modulation in inhibitory control paradigm. Data were collected in 19 right-handed volunteers with mean age of 21.21 $( \pm 1,77)$ years who performed the Go/NoGo paradigm for 50 minutes, with synchronized electroencephalogram recording to obtain the ERPs. The time on task effect induced significant changes in subjective variable, cognitive performance and maximum amplitudes of the N2 and P3 component. Our results suggest that when our brain is subjected to extensive cognitive demands, there are adaptations to maintain the behavioral performance through strategy reallocation of energy resources.
\end{abstract}

KEYWORDS: long time task, inhibitory control, ERP, N2, P3

Ambientes contemporâneos caracterizados pelo ritmo acelerado da rotina de trabalho submetem grande parte da população a uma complexa demanda cognitiva por longos períodos de tempo (Murata, Uetake, \& Takasawa, 2005; Park, Kim, Chung, \& Hisanaga, 2001). Essa rotina é repleta de estímulos concorrentes à nossa capacidade de processamento perceptivo, exigindo uma rápida adaptação da seleção de informações relevantes para se obter um bom desempenho nas atividades (Benikos, Johnstone, \& Roodenrys, 2013; Chernyshev, Lazarev, Bryzgalov, \& Novikov, 2015).

Quando realizada diariamente, sem períodos de recuperação adequados, essa rotina pode levar ao prejuízo físico e mental (Melina, Keller, \& Coelho, 2013; Pereira, Salles, \& Passos, 2010; Quirino \& Villemor-Amaral, 2015), já que a interação eficiente em contextos hiperestimulados necessita de ajustes constantes no escopo atencional (Friedman-Hill, Robertson, Desimone, \& Ungerleider, 2003; Shomstein, 2012) e processamentos cognitivos complexos exigem grande gasto metabólico para o cérebro (Gergelyfi, Jacob, Olivier, \& Zénon, 2015; Ishii, Tanaka, \& Watanabe, 2014; van der Linden, Frese, \& Meijman, 2003).

Devido a esse aumento no gasto energético do cérebro, a execução de processamentos cognitivos repetidos, por tempo prolongado (Hopstaken, Linden, Bakker, \& Kompier, 2015; Lal \& Craig, 2002), somado a baixas recompensas (Boksem

\footnotetext{
*E-mail: hmurilodemelo@gmail.com

- Submetido: 10/05/2016; Revisado: 18/04/2017; Aceito: 19/06/2017.
} 
\& Tops, 2008), resulta no abandono do comportamento que está sendo realizado (Boksem, Meijman, \& Lorist, 2005; Phillips, 2015), podendo acarretar em comportamentos falhos ou acidentes (Borghini, Astolfi, Vecchiato, Mattia, \& Babiloni, 2014).

Diante desse contexto, encontraram-se evidências comportamentais que sugerem um prejuízo cognitivo durante a realização de uma tarefa por tempo prolongado, como em um experimento no qual participantes foram submetidos a uma tarefa de atenção seletiva, durante duas horas, em que se observou queda significativa de desempenho conforme o desenvolvimento da tarefa (Faber, Maurits, \& Lorist, 2012). Efeito semelhante foi encontrado em estudos que utilizaram tarefas de atenção seletiva (Boksem et al., 2005; Wascher et al., 2014) e simuladores de direção (Ting, Hwang, Doong, \& Jeng, 2008; Zhao, Zhao, Liu, \& Zheng, 2012).

Considerando a dinâmica e o grande volume de estímulos encontrados na rotina contemporânea, além dos processos atencionais, observa-se a importância da inibição de comportamentos pré-planejados durante uma rápida mudança de contexto em prol de estratégias mais eficazes, através do processo de controle inibitório (Kato, Endo, \& Kizuka, 2009; Lage et al., 2011; Murata et al., 2005). Entre os paradigmas experimentais de avaliação desse processo cognitivo, a tarefa Go/NoGo é comumente utilizada. Durante o protocolo, o indivíduo deve interromper uma resposta pré-estabelecida em prol da presença de um estímulo alvo que requer um padrão de resposta diferente (Aron, Robbins, \& Poldrack, 2014; Pandey et al., 2012).

O processo de inibição comportamental no paradigma Go/NoGo tem sido estudado por potenciais relacionados a eventos (sigla em inglês, ERPs; Bokura, Yamaguchi, \& Kobayashi, 2001; Menon, Adleman, White, Glover, \& Reiss, 2001). Para a obtenção dos ERPs, o sinal de EEG é sincronizado com a apresentação de um estímulo na tela do computador e são extraídos componentes com latências e amplitudes cujas propriedades refletem a despolarização de um grupo de neurônios de regiões específicas do cérebro (Brandeis \& Lehmann, 1986; Kok, 1997).

Nesse paradigma, destacam-se a modulação nos componentes N2 e P3 (Falkenstein, Hoormann, \& Hohnsbein, 1999; Jonkman, 2006; Kaiser et al., 2003; Smith, Smith, Provost, \& Heathcote, 2010). O componente N2 está relacionado ao processamento atencional após a identificação do estímulo, verificando sua relevância para a execução do comportamento relacionado à tarefa (Boksem et al., 2005; Folstein \& Van Petten, 2007; Lange , Wijers, Mulder, \& Mulder, 1998). Esse componente também é associado ao controle da resolução de conflitos entre estímulos concorrentes, refletindo o processo de inibição de uma resposta programada em prol de outra estratégia (Falkenstein et al., 1999; Pandey et al., 2012).

O papel do componente $\mathrm{P} 3$ relaciona-se com a atualização de contexto da memória de trabalho, atuando após o processo atencional de comparação de estímulos. Ele possui a função de armazenar a informação por um curto período de tempo, caso o estímulo tenha se alterado e exija uma resposta comportamental (Brydges, Fox, Reid, \& Anderson, 2014; Polich, 2007). Sendo assim, os ERPs tornam-se uma importante ferramenta para investigar alterações no funcionamento cerebral durante a execução de tarefas cognitivas (Sheppard \& Vernon, 2008; Woods, Wyma, Yund, Herron, \& Reed, 2015; Wronka, Kaiser, \& Coenen, 2013).

As alterações subjetivas e de desempenho cognitivo decorrentes de demandas cognitivas prolongadas já são comumente reportadas na literatura científica, portanto, este trabalho pretende acrescentar evidências eletrofisiológicas através de ERPs, com o objetivo de fornecer insights sobre possíveis mecanismos neurofisiológicos que estão relacionados ao processo de queda de desempenho observado durante a indução de cargas mentais por tempo prolongado. Para tal, este estudo pretende investigar alterações na autopercepção, no desempenho cognitivo e em parâmetros eletrofisiológicos durante a realização do paradigma Go/NoGo por tempo prolongado.

\section{MÉTODO}

\section{Participantes}

Participaram do experimento 19 voluntários (9 homens), destros, com idade entre 18 e 24 anos $(\mathrm{M}=21,21 \mathrm{DP}=$ 1,77 anos). Todos os participantes tinham visão normal ou corrigida por lentes. O termo de consentimento livre e esclarecido (TCLE) foi assinado por todos antes do início do experimento, em conjunto com um questionário sobre seu estado físico e mental. Os participantes que informaram: (a) ser um atleta (ter um ciclo de treinamento para competição); (b) ser praticante de meditação regular por mais de um ano; (c) possuir vínculo empregatício noturno; (d) ter histórico de abuso de drogas ou medicamentos psicoativos; (e) possuir distúrbios neurológicos ou psicológicos; (f) ingerir cafeína, bebidas energéticas, cigarro, bebidas alcoólicas $24 \mathrm{~h}$ antes do experimento; (g) praticar exercício físico intenso (fora da rotina) $24 \mathrm{~h}$ antes do experimento e (h) ter restrição ou privação de sono na noite anterior foram excluídos da amostra. Este estudo foi realizado após a aprovação do Comitê de Ética e Pesquisa com Seres Humanos da UFSC (CEPSH), sob parecer número CAAE: 44053615.4.0000.0121. 


\section{Materiais}

Setting experimental. O experimento foi realizado em um ambiente com iluminação artificial constante (lâmpada fluorescente tubular de $14 \mathrm{~W}$, deixando o ambiente com luminosidade média de 250 LUX), em uma sala com divisórias com isolamento acústico (com ruído sonoro entre $35-45 \mathrm{~dB}$ ), que possuía divisória para separar experimentador e participante durante a execução da tarefa. Os participantes foram confortavelmente posicionados em uma cadeira, ficando a $90 \mathrm{~cm}$ de distância de um monitor de 21 " LCD, ajustado na altura dos olhos, em que eram apresentados os estímulos.

Registro de EEG. O registro de EEG foi realizado com um amplificador, da marca Mitsar ${ }^{\circledR}$, utilizando 19 canais, através da touca EEGCap (sistema internacional 10-20), nas regiões: Fp1, Fp2, F7, F3, Fz, F4, F8, T3, C3, Cz, C4, T6, T7, P3, Pz, P4, T8, O1 e O2. Todos os canais utilizaram referência monopolar através de eletrodos auriculares (A1 e A2). Foi realizado o registro do eletroculograma (EOG) no olho esquerdo para a realização da correção de artefatos por movimento ocular através da técnica da análise de componente independente, conforme descrito por Jung et al. (2000). A impedância dos eletrodos não ultrapassou $10 \mathrm{k} \Omega$. A taxa de amostragem foi de $500 \mathrm{~Hz}$ e durante o registro foi utilizado o filtro passa banda de $0,1 \mathrm{~Hz}$ a $100 \mathrm{~Hz}$ e o filtro notch na faixa de $60 \mathrm{~Hz}$.

\section{Tarefa Cognitiva e Procedimento Experimental}

A tarefa utilizada baseou-se no paradigma Go/NoGo, programada no software PsyTask ${ }^{\circledR}$, contendo cinco blocos com 250 tentativas, com intervalos de 30 segundos para descanso entre os blocos, tendo 50 minutos de duração. Cada estímulo era precedido por uma cruz de fixação $(200 \mathrm{~ms})$, seguido de um tempo de espera $(800 \mathrm{~ms})$ até a apresentação do estímulo (200ms). Após o estímulo, o participante tinha intervalo $(800 \mathrm{~ms})$ para realizar a resposta, em seguida iniciava-se uma nova tentativa (Figura 1).

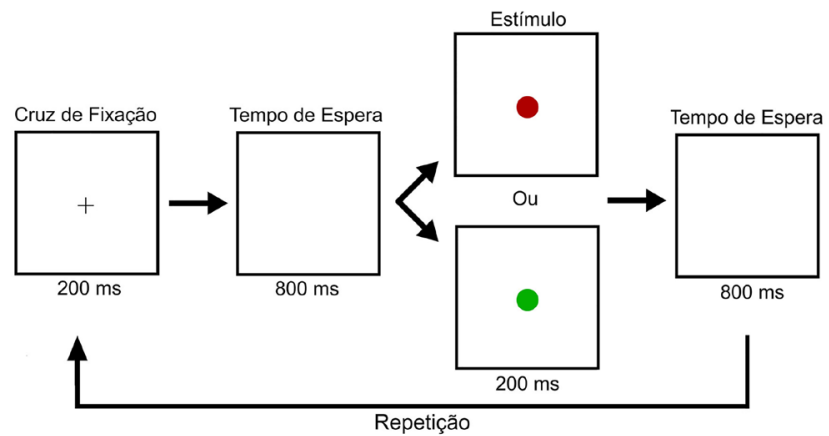

Figura 1. Fluxograma da apresentação de estímulos. Em nosso protocolo, a cruz de fixação é seguida de um círculo verde ou vermelho solicitando a resposta do participante de acordo com a cor do estímulo.
A apresentação dos tipos de estímulos era pseudoaleatória, com a porcentagem de frequência diferenciada, sendo: estímulos alvo (20\%) e estímulos não-alvo $(80 \%)$. O estímulo alvo era representado por um círculo verde, o não-alvo vermelho, ambos com $2 \mathrm{~cm}$, posicionados no centro da tela. Para responder a tarefa, o participante deveria apertar o botão esquerdo do mouse durante a apresentação dos estímulos alvo (círculo verde) e o botão direito para os estímulos não-alvo (círculo vermelho).

Realizava-se um período de habituação à tarefa com 50 tentativas, para verificar se não havia dúvidas sobre o procedimento. Em seguida, caso não houvesse questionamentos, dava-se início ao experimento. Durante todos os intervalos entre os blocos, foi solicitado ao participante respostas subjetivas relacionadas à autopercepção de atenção, sonolência e motivação em relação à continuação da tarefa, quantificando seu estado mental através de notas com a variação de um a dez.

\section{Extração de Dados e Análise}

Desempenho cognitivo. Foram analisados: (a) Tempo de Reação do Estímulo Alvo (TR EA); (b) Tempo de Reação do Estímulo Não-Alvo (TR ENA); (c) Número de Erros por Omissão (EO) (não pressionar nenhum botão durante o período de resposta) ; (d) Número de Erros por Comissão em Estímulos Alvo (ECA) (pressionar o botão errado em relação ao estímulo apresentado); (e) Número de Erros por Comissão em Estímulos Não-Alvo (ECNA) (pressionar o botão errado em relação ao estímulo apresentado) e (f) Tempo de Discriminação (TD) (calculado através da diferença entre TR EA e TR ENA). Os dados foram agrupados utilizando o primeiro e o último bloco como critério de seleção, sendo consideradas apenas as respostas corretas entre 200 e $800 \mathrm{~ms}$ após os estímulos para o cálculo do TR EA e TR ENA de cada bloco.

O processamento de sinais e a extração dos ERPs foram realizados na toolbox EEGLab 13.5.4b (Delorme et al., 2011), utilizando o Matlab versão 2013a. Os dados foram submetidos a um filtro off-line passa banda de 0,5 $35 \mathrm{~Hz}$, tendo os artefatos de movimento ocular e muscular corrigidos através do método de análise de componente independente (Jung et al., 2000): Os trechos que continuaram comprometidos por artefato de movimento ocular foram retirados da análise. Para a análise dos ERPs, excluiu-se as tentativas em que o participante errou a resposta. Foram extraídas épocas de um segundo $(-100 \mathrm{~ms}$ à $900 \mathrm{~ms})$, utilizou-se o período de $100 \mathrm{~ms}$ precedentes à apresentação do estímulo como parâmetro para a correção de linha de base. Os dados foram agrupados de acordo com o tipo de estímulo (alvo e não-alvo) e período da tarefa (primeiro e último bloco).

Análise estatística. Os parâmetros subjetivos e as variáveis de desempenho cognitivo foram submetidos à estatística descritiva e ao teste de normalidade Shapiro- 
Wilk (Disponíveis no Documento Suplementar 1), através do Software Stata versão 14.0. O teste t de Student para amostras pareadas foi utilizado para comparar essas variáveis durante o primeiro e último bloco, atribuindo-se o intervalo de confiança de $95 \%$.

A amplitude máxima do componente N2 (no intervalo de $200-300 \mathrm{~ms}$ ) e P3 (no intervalo de $250-400 \mathrm{~ms}$ ) para os estímulos alvo e não-alvo foram extraídos na toolbox
EEGLab 13.5.4b (Delorme et al., 2011). Para verificar o efeito do tempo de realização da tarefa, as amplitudes do primeiro e último bloco foram comparadas através do teste t de Student para amostras pareadas, sendo realizado um teste para cada tipo de estímulo (alvo e não-alvo). Também foi realizada uma correlação de Pearson entre as amplitudes máximas do componente $\mathrm{N} 2$ e $\mathrm{P} 3$, variáveis subjetivas e de desempenho cognitivo.

\section{RESULTADOS}

A comparação entre as variáveis de autorrelato no primeiro e último bloco, realizada através do teste $t$ de Student para amostras pareadas (Tabela 1), sugere que os escores de Autopercepção de Atenção (AT) $[t(18)=8,11$, $p=0,0001]$ e Autopercepção de Motivação (AM) [t (18) $=7,34, p=0,0001]$ apresentaram redução significativa no último bloco ( $\mathrm{AT}=3,05, \mathrm{AM}=2,73)$, quando comparado ao primeiro ( $\mathrm{AT}=6,94, \mathrm{AM}=6,52$ ). $\mathrm{O}$ resultado inverso foi observado na Autopercepção de Sonolência (AS), em que se observou um aumento significativo $[t(18)=-4,48$, $p=0,0001]$ quando comparados o primeiro $(\mathrm{M}=5,15) \mathrm{e}$ último bloco $(\mathrm{M}=7,68)$.

Os resultados do teste $t$ de Student na comparação das variáveis de desempenho cognitivo nos dois períodos da tarefa (Tabela 1) sugerem que o Tempo de Reação de Estímulos Alvo (TR EA) $[t(18)=-3,46, p=0,004]$ e o Tempo de Discriminação de Estímulos (TD) $[t(18)=-6,88, p$ $=0,0001]$ apresentaram um aumento significativo no último bloco (TR EA = 399,73, TD = 124,00), quando comparado ao primeiro ( $\mathrm{TR} \mathrm{EA}=375,21, \mathrm{TD}=73,32)$. A medida de velocidade de resposta relacionada ao Tempo de Reação para Estímulos Não-Alvo (TR ENA) sofreu uma redução significativa $[t(18)=3,01, p=0,043]$ no último bloco (M $=275,73)$, quando comparado ao primeiro $(\mathrm{M}=301,89)$.

As variáveis relacionadas à frequência de erros cometidos durante a tarefa também apresentaram alterações significativas, quando comparados o primeiro e último bloco da tarefa. O número de Erros por Omissão (EO) $[t$ $(18)=2,40, p=0,032]$ e o número de Erros por Comissão em Estímulos Alvo (ECA) [ $t(18)=2,94, p=0,008$ ] apresentaram um aumento significativo no último bloco. Não foram observadas diferenças significativas entre o número de Erros por Comissão em Estímulos Não-Alvo (ECNA) [ $t$ $(18)=0,75, p=0,46]$ quando comparados o primeiro $(\mathrm{M}=$ $1,26)$ e último bloco $(\mathrm{M}=0,84)$.

A realização de uma tarefa de longa duração provocou alteração significativa na modulação dos ERPs de estímulos alvo (Figura 2A). Os resultados do teste $t$ de Student sugerem que a comparação da média da amplitude máxima do componente $\mathrm{N} 2$ no primeiro $(\mathrm{M}=-1,97)$ e último $(\mathrm{M}=-5,28)$

Tabela 1

Resultados do teste t de Student para a comparação das variáveis de autopercepção, desempenho cognitivo e ERPs durante o primeiro e último bloco.

\begin{tabular}{lcccc}
\hline $\begin{array}{l}\text { Variáveis } \\
\text { (N=19) }\end{array}$ & $\begin{array}{c}\text { Média do } \\
\text { Primeiro Bloco (DP) }\end{array}$ & $\begin{array}{c}\text { Média do } \\
\text { Último Bloco (DP) }\end{array}$ & $\boldsymbol{t}$ & $\boldsymbol{p}$ \\
\hline AT*** & $6,94(1,74)$ & $3,05(1,95)$ & 8,11 & 0,0001 \\
AS*** & $5,15(2,24)$ & $7,68(1,33)$ & 4,48 & 0,0001 \\
AM*** & $6,52(2,45)$ & $2,73(2,35)$ & 7,34 & 0,0001 \\
TR EA** & $375,21(52,88)$ & $399,73(47,82)$ & 3,46 & 0,004 \\
TR ENA* & $301,89(49,69)$ & $275,73(36,83)$ & 3,01 & 0,043 \\
ECA** & $4,42(3,79)$ & $7,05(5,70)$ & 2,94 & 0,008 \\
ECNA & $1,26(2,25)$ & $0,84(0,95)$ & 0,75 & 0,46 \\
EO* & $2,94(3,86)$ & $6,94(8,99)$ & 2,40 & 0,032 \\
TD*** & $73,32(34,44)$ & $124,00(38,06)$ & 6,88 & 0,0001 \\
N2 EA** & $-1,97(4,38)$ & $-5,28(5,69)$ & 3,05 & 0,009 \\
P3 EA & $12,18(4,25)$ & $12,80(4,01)$ & 0,77 & 0,29 \\
N2 ENA & $1,08(3,23)$ & $1,78(3,01)$ & 0,41 & 0,83 \\
P3 ENA** & $7,21(3,57)$ & $5,09(4,02)$ & 3,92 & 0,001 \\
\hline
\end{tabular}

Legenda. $\mathrm{DP}$ = Desvio Padrão; $\mathrm{AT}$ = Autopercepção de atenção; $\mathrm{AS}=$ Autopercepção de sonolência; $\mathrm{AM}=$ Autopercepção de Motivação na realização da tarefa; TR EA = Tempo de Reação dos Estímulos Alvo; TR ENA = Tempo de Reação dos Estímulos Não-Alvo; ECA = Erros por Comissão em Estímulos Alvo; ECNA = Erros por Comissão em Estímulos Não-Alvo; EO = Erros por Omissão; TD = Tempo de discriminação entre os estímulos; ${ }^{*} \mathrm{p}<0,05 ; * * \mathrm{p}<0,005 ; * * * \mathrm{p}<0,0001$. 
bloco da tarefa apresenta um aumento significativo $[t(18)=$ $3,05, p=0,009]$. Não foi observada alteração significativa [ $t$ $(18)=0,77, p=0,29]$ na comparação do componente $\mathrm{P} 3$ no início $(\mathrm{M}=12,18)$ e final da tarefa $(\mathrm{M}=12,80)$.

$\mathrm{O}$ resultado da comparação entre o primeiro e último bloco para estímulos não-alvo (Figura $2 \mathrm{~B}$ ) sugere que o componente $\mathrm{P} 3$ apresentou uma redução significativa $[t$ $(18)=3,92, p=0,001]$, quando comparado à média da amplitude máxima dos participantes durante o primeiro $(\mathrm{M}=7,21)$ e último $(\mathrm{M}=5,09)$ bloco da tarefa. O resultado do teste $t$ de Student não encontrou diferença significativa entre as amplitudes do componente $\mathrm{N} 2[t(18)=0,41$, $p=0,83]$ durante os dois períodos da tarefa.
A correlação de Pearson entre as variáveis eletrofisiológicas, subjetivas e de desempenho cognitivo (Tabela 2) sugere que, para estímulos alvo, há um coeficiente de correlação moderado entre a amplitude máxima do componente N2 e o TR EA $[r=0,49, \mathrm{p}=0,007]$. Nessa análise, observou-se que os valores de significância para a correlação entre $\mathrm{N} 2$ e ECA apresentaram valores de $p$ próximos da significância $[r=-0,43, p=0,07]$. Para os estímulos não-alvo, os resultados da correlação de Pearson sugerem associações moderadas entre as variáveis $\mathrm{N} 2 \mathrm{e}$ $\mathrm{ECNA}[r=-0,33, p=0,03], \mathrm{P} 3$ e ECNA $[r=-0,40, p=0,01]$ e P3 e AT $[r=0,35, p=0,03]$.
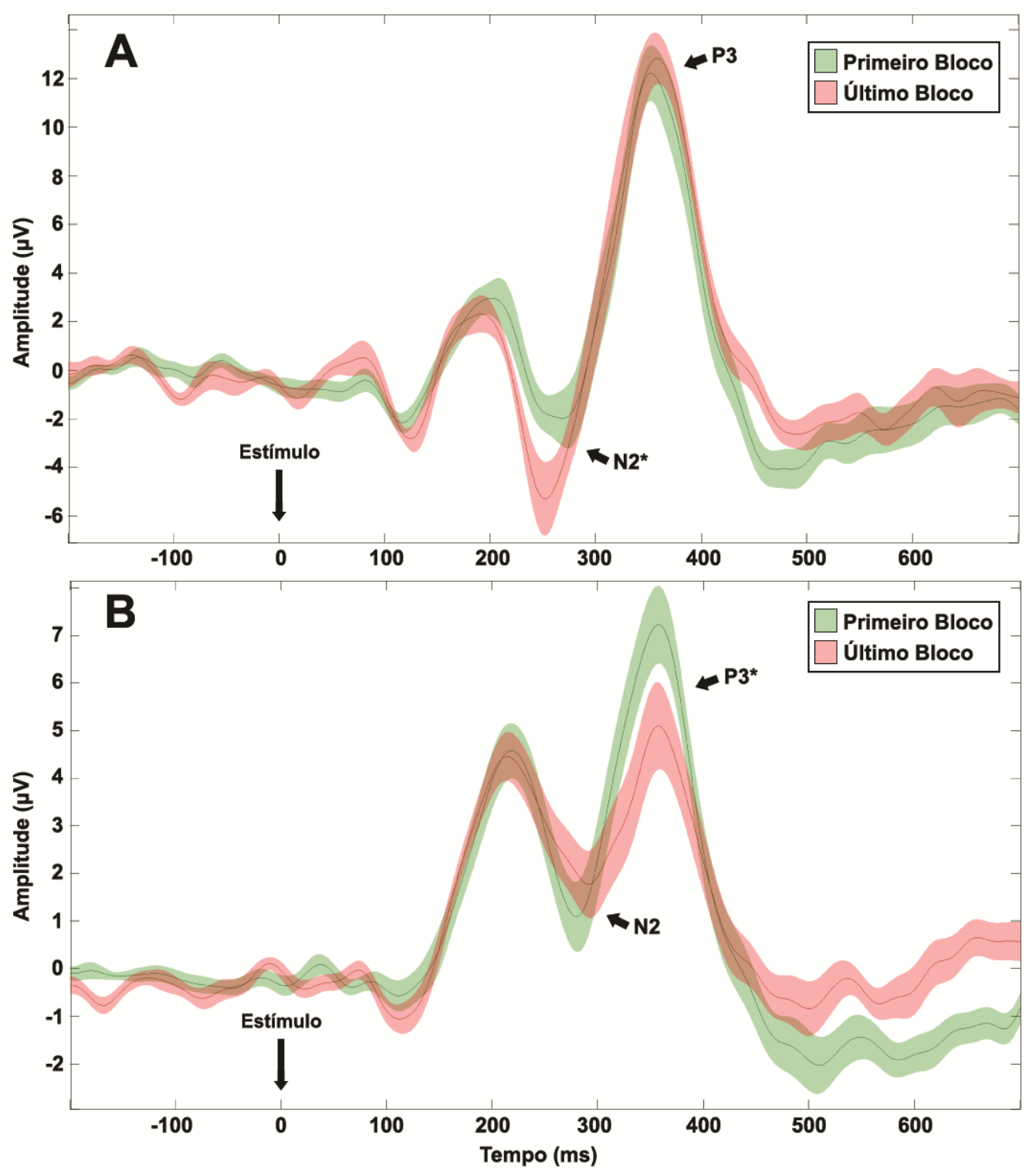

Figura 2. Comparação da média dos ERPs dos durante o primeiro e último bloco da tarefa para estímulos alvo (A) e não-alvo (B). A linha verde representa a Média dos ERPs dos participantes durante o primeiro bloco e seu contorno indica os valores do desvio padrão. A linha vermelha representa a média dos ERPs dos participantes durante o último bloco e seu contorno indica os valores do desvio padrão. *p<0,05. 
Tabela 2

Resultados significativos da correlação entre os componentes N2 e P3, variáveis subjetivas e de desempenho cognitivo.

\begin{tabular}{|c|c|c|c|c|}
\hline \multirow{2}{*}{$\begin{array}{l}\text { Estímulo } \\
\text { Alvo }\end{array}$} & \multicolumn{2}{|c|}{ Variáveis } & \multirow{2}{*}{$\begin{array}{c}\boldsymbol{r} \\
-0,34\end{array}$} & \multirow{2}{*}{$\begin{array}{c}p \\
0,073\end{array}$} \\
\hline & $\mathrm{N} 2$ & ECA & & \\
\hline & $\mathrm{N} 2$ & TR EA & 0,49 & $0,0076^{*}$ \\
\hline \multirow[t]{3}{*}{ Não-Alvo } & $\mathrm{N} 2$ & ECNA & $-0,33$ & $0,039^{*}$ \\
\hline & P3 & ECNA & $-0,40$ & $0,011^{*}$ \\
\hline & P3 & AT & 0,35 & $0,030^{*}$ \\
\hline
\end{tabular}

Legenda. AT = Autopercepção de atenção; TR EA = Tempo de Reação dos Estímulos Alvo; ECA = Erros por Comissão em Estímulos Alvo; ECNA = Erros por Comissão em Estímulos Não-Alvo; EO = Erros por Omissão; N2 = Amplitude máxima do componente N2; P3 = Amplitude máxima do componente $\mathrm{P} 3 ;{ }^{*} \mathrm{p}<0,05 ;{ }^{* *} \mathrm{p}<0,005 ; * * * \mathrm{p}<0,0001$.

\section{DISCUSSÃO}

Neste experimento, observou-se que medidas baseadas em construtos psicológicos relacionados a autopercepção de atenção, sonolência e motivação possuem sensibilidade para detectar alterações em estados psicológicos durante a realização de uma tarefa por tempo prolongado. Resultado semelhante foi observado em um experimento que submeteu os participantes à realização de tarefas cognitivas durante três horas e observaram redução na autopercepção da qualidade atencional (Boksem et al., 2005). Outra pesquisa que utilizou tarefas prolongadas em simuladores de direção por tempo prolongado também encontrou um aumento significativo na percepção de sonolência por meio do autorrelato dos participantes (Ting et al., 2008).

Além dessas pesquisas, outros experimentos também corroboram os achados do presente trabalho (Faber et al., 2012; Kato et al., 2009), sugerindo que respostas subjetivas podem ser uma fonte de dados fidedigna para detectar modulações em estados psicológicos relacionados à realização de tarefas por tempo prolongado. Embora alguns pesquisadores (Fairclough \& Venables, 2006) indiquem alta correlação entre relatos subjetivos e alterações eletrofisiológicas, nosso experimento encontrou apenas uma correlação moderada entre a percepção de atenção e a amplitude máxima do componente P3 em estímulos não-alvo, enquanto os outros constructos não apresentaram coeficientes de correlação significativos.

De fato, a amplitude do componente $\mathrm{P} 3$ possui uma estrita relação com a qualidade do funcionamento cognitivo exibido por um indivíduo (Brydges et al., 2014; Polich, 2007) e a restrição da correlação deste componente com um indicador autopercepção de qualidade atencional confirma a ênfase de alguns autores de que esse constructo possui boa sensibilidade para detectar alterações durante cargas cognitivas prolongadas (Faber et al., 2012; Kato et al., 2009). Nesse caso, o indicador subjetivo de qualidade atencional possui um grau moderado de relação com alterações neurofisiológicas, o que aumenta a fidedignidade desse constructo para avaliação de carga mental.

Por outro lado, a ausência de correlação dos outros constructos avaliados pode ter relação com a simplicidade das medidas utilizadas neste experimento, em que a complexidade de alterações envolvidas na modulação de motivação e sonolência pode não ter sido contemplada apenas com uma única pergunta. Escalas tradicionalmente utilizadas para mensurar carga mental (Hart \& Stavenland, 1988) têm uma aplicação longa, o que diminui a precisão do momento em que a carga mental é induzida, fazendo com que esse intervalo possa servir para descanso e cause alterações nas respostas. Sendo assim, o desenvolvimento de escalas breves e de alta confiabilidade se torna um desafio para pesquisadores da área de carga mental.

O principal processo cognitivo observado através do paradigma Go/NoGo está relacionado ao processo de inibição das respostas automatizadas (Dalley, Everitt, \& Robbins, 2011; Hartmann, Sallard, \& Spierer, 2015). Os resultados deste trabalho sugerem que quando realizado por um período prolongado, sem um período de recuperação adequado, a qualidade da habilidade de controle inibitório apresenta um prejuízo significativo, seja em relação ao tempo de resposta ou número de erros.

Resultados semelhantes foram reportados em experimentos que utilizaram o paradigma Go/NoGo com uma hora (Kato et al., 2009) e três horas (Murata et al., 2005) de duração, em que os participantes apresentaram prejuízo significativo no TR EA e no número de erros de associação entre estímulo resposta, quando comparados os valores dos blocos ao longo da tarefa. Assim como os resultados reportados através do paradigma Go/NoGo, outros experimentos utilizando tarefas relacionadas à atenção seletiva (Faber et al., 2012) e tempo de reação (Ting et al., 2008) sugerem que o prejuízo de desempenho observado em tarefas de demanda cognitiva prolongada pode ser extrapolado para outros processos cognitivos.

Devido à ausência de informações sobre as alterações em estímulos não-alvo e no número de erros de comissão alvo e não-alvo separadamente, este trabalho agrega novas evidências relacionadas aos processos de adaptação do cérebro durante uma demanda cognitiva prolongada. A hipótese comumente compartilhada pela comunidade científica sugere que durante demandas prolongadas ocorre 
uma realocação de recursos cognitivos para a execução de processos automatizados, gerando um prejuízo de desempenho em tarefas que exigem controle cognitivo via mecanismo top-down (Borghini et al., 2014; Lorist et al., 2000, 2008).

A redução no tempo de resposta de estímulos não-alvo observada neste experimento pode ser interpretada como um prejuízo comportamental também, já que respostas automatizadas mais rápidas podem prejudicar o processo de inibição de padrões comportamentais pré-estabelecidos. Sendo assim, o aumento de velocidade de respostas automatizadas e o prejuízo no tempo de reação para inibição corroboram com a hipótese de que os prejuízos cognitivos relacionados a demandas mentais prolongadas afetam principalmente os mecanismos que necessitam de controle cognitivo, sugerindo que a facilitação na execução de processos automatizados pode colaborar significativamente nesse prejuízo.

A análise dos ERPs permite mensurar a sincronização da atividade de neurônios recrutados de uma região específica do cérebro relacionada ao processo cognitivo requerido, a variação de sua amplitude pode indicar a mobilização de recurso energético ou mecanismos de eficiência do sistema durante a realização de processamentos cognitivos (Kok, 1997, 2001). Neste estudo, encontrou-se influência significativa do período de realização da tarefa no aumento do componente $\mathrm{N} 2$ para estímulos alvo e na redução do componente $\mathrm{P} 3$ de estímulos não-alvo.

Pesquisadores têm reportado (Grabner, Fink, Stipacek, Neuper, \& Neubauer, 2004; Neubauer \& Fink, 2003, 2009) que processos de aprendizagem e adaptação a uma tarefa provocam mecanismos de adaptação eletrofisiológica relacionados à eficiência neural. Esse modelo (Neubauer \& Fink, 2009) sugere que, após um período de habituação e facilitação da execução de uma tarefa cognitiva, há uma redução na atividade cortical relacionada à tarefa, tornando o sistema mais eficiente durante a execução desse processo.

$\mathrm{O}$ aumento do componente N2 observado neste experimento pode ter relação com um incremento de energia necessário para desempenho da sua função, dificultando processos de habituação à tarefa, que, nesta pesquisa, pode ser refletido pelo aumento no tempo de reação de estímulos alvo. Sendo assim, durante a realização de uma tarefa por tempo prolongado, há um aumento na energia necessária para a manutenção de processos cognitivos atencionais relacionados ao componente $\mathrm{N} 2$, porém, esse aumento de amplitude não está relacionado com qualidade, tornando-se apenas um maior gasto energético para continuar executando a tarefa.

A alteração na amplitude do componente $\mathrm{P} 3$ pode ser compreendida através de sua relação com a produção de processos cognitivos conscientes (Babiloni, 2005; Rutiku, Martin, Bachmann, \& Aru, 2015), sendo observada, neste experimento, através de sua correlação com a autopercepção de qualidade atencional. Compreende-se que a redução na energia despendida para a execução do $\mathrm{P} 3$ está relacionada à realocação de recursos energéticos da execução de processos cognitivos conscientes para processos perceptuaisatencionais pré-conscientes, como os relacionados ao componente N2. Nesse sentido, a adaptação do cérebro durante tarefas de longa duração pode ser caracterizada como uma alteração na prioridade de recursos energéticos entre processos cognitivos conscientes para pré-conscientes.

A hipótese de realocação de recursos pode ser compreendida através da característica de automatização das tarefas de longa duração, em que o recurso energético relacionado a processos cognitivos conscientes é mobilizado para a execução de outros processos cognitivos, não necessariamente garantindo a melhora do desempenho, mas impedindo que a execução da tarefa seja interrompida. Em um experimento semelhante, em que 18 participantes realizaram o paradigma Go/NoGo durante 60 minutos ininterruptos, foram extraídos ERPs em três blocos de 20 minutos, porém não foram encontradas alterações significativas nas amplitudes dos componentes N2 e P3, apenas nas latências (Kato et al., 2009).

A divergência dos resultados entre os trabalhos pode ser explicada por diferenças individuais de mecanismos de adaptabilidade à carga mental, por parte da amostra do experimento que aumenta a variação dos resultados obtidos por meio dos ERPs, o que influenciaria na ausência de significância estatística. Outro fator importante está relacionado com a existência de blocos durante a tarefa, que, diferentemente de uma tarefa ininterrupta, pode ter fornecido um período breve de recuperação entre os blocos, que pode sugerir que o experimentou não investigou o estado mental de tempo prolongado, mas sim de fadiga mental.

A discriminação entre estes dois processos mentais está vinculada à disponibilidade de recursos energéticos disponíveis para uso, que é ausente durante a manifestação de fadiga mental, já que experimentos sugerem que há uma redução na amplitude do componente N2 após a realização de tarefas durante duas ou três horas ininterruptas em paradigmas de atenção seletiva (Boksem et al., 2005; Faber et al., 2012). Hipotetiza-se que devido ao aumento no gasto energético para a manutenção dos comportamentos em tarefas por tempo prolongado, sem um tempo de recuperação satisfatório, pode ativar mecanismos de fadiga sináptica (como o esgotamento de neurotransmissores) e iniciar a manifestação de sintomas de fadiga mental. Nesse sentido, os resultados eletrofisiológicos observados em Kato et al. (2009) podem estar relacionados a esse período de transição, pois sua tarefa não tem um tempo prolongado tão alto como os protocolos tradicionais de fadiga mental (Borghini et al., 2014; Wascher et al., 2014), porém não tem períodos de recuperação adequados (Lal \& Craig, 2002).

Sendo assim, este trabalho fomenta a utilização de tecnologias de mapeamento cerebral na avaliação de processos psicológicos, devido ao seu aprimoramento do processo de aquisição de dados, uma vez que fenômenos 
semelhantes em relação aos sinais comportamentais (prejuízo no desempenho cognitivo e redução em indicadores de autopercepção) podem ser compreendidos com maior profundidade, como é o caso da discriminação entre fadiga mental e tarefas com tempo prolongado. Além disso, informações eletrofisiológicas podem fornecer insights para o desenvolvimento de hipóteses relacionadas às complexidades do comportamento humano.

Os resultados deste trabalho podem colaborar com o desenvolvimento de tecnologias aplicáveis a nível stricto sensu, através do desenvolvimento de sensores que integram informações eletrofisiológicas com medidas psicológicas tradicionais para aprimorar a precisão do reconhecimento de diferentes estados mentais, conforme proposto em Hsu, Wang, Chen, \& Chen, 2015. Cabe ressaltar a limitação do estudo relacionada ao número de participantes da pesquisa, cujo número não possibilitou a criação de grupos homogêneos a partir dos diferentes níveis de comprometimento de desempenho durante a indução de carga mental prolongada. Especula-se que, para estudos futuros, seja interessante investigar a influência de medidas de resistência psicológica na modulação das respostas durante a indução de cargas mentais prolongadas, bem como possíveis correlatos eletrofisiológicos dessas diferenças individuais.

\section{CONCLUSÃO}

Os resultados deste estudo sugerem que a execução da atividade de inibição comportamental (Go/NoGo) por tempo prolongado provoca prejuízos no desempenho cognitivo e alterações nos parâmetros de autopercepção relacionados à atenção, sonolência e motivação em continuar realizando a tarefa. A análise de ERPs fornece evidências indicando que, durante a realização de tarefas com duração prolongada, nosso cérebro tende a priorizar a distribuição de recursos energéticos para processos essenciais para a execução da tarefa, realocando recursos energéticos de processos cognitivos conscientes para processos atencionais pré-conscientes, devido à característica de automatização de tarefas prolongadas. Essa alteração parece não possuir relação com o retorno de desempenho das respostas, apenas garante que a atividade continue sendo executada.

\section{REFERÊNCIAS}

Aron, A. R., Robbins, T. W., \& Poldrack, R. A. (2014). Inhibition and the right inferior frontal cortex: One decade on. Trends in Cognitive Sciences, 18(4), 177-185. doi: 10.1016/j. tics.2013.12.003

Babiloni, C. (2005). Visuo-spatial consciousness and parietooccipital areas: A high-resolution EEG study. Cerebral Cortex, 16(1), 37-46. doi: 10.1093/cercor/bhi082

Benikos, N., Johnstone, S. J., \& Roodenrys, S. J. (2013). Varying task difficulty in the Go/Nogo task: The effects of inhibitory control, arousal, and perceived effort on ERP components. International Journal of Psychophysiology, 87(3), 262-272. doi: 10.1016/j. ijpsycho.2012.08.005

Boksem, M. a S., Meijman, T. F., \& Lorist, M. M. (2005). Effects of mental fatigue on attention: An ERP study. Brain Research. Cognitive Brain Research, 25, 107-116. doi: 10.1016/j. cogbrainres.2005.04.011

Boksem, M. a S., \& Tops, M. (2008). Mental fatigue: Costs and benefits. Brain Research Reviews, 59(1), 125-139. doi: 10.1016/j.brainresrev.2008.07.001

Bokura, H., Yamaguchi, S., \& Kobayashi, S. (2001). Electrophysiological correlates for response inhibition in a Go/NoGo task. Clinical Neurophysiology, 112(12), 2224-2232. doi. $10.1016 / \mathrm{S} 1388-2457(01) 00691-5$

Borghini, G., Astolfi, L., Vecchiato, G., Mattia, D., \& Babiloni, F. (2014). Measuring neurophysiological signals in aircraft pilots and car drivers for the assessment of mental workload, fatigue and drowsiness. Neuroscience and Biobehavioral Reviews, 44, 58-75. doi: 10.1016/j.neubiorev.2012.10.003

Brandeis, D., \& Lehmann, D. (1986). Event-related potentials of the brain and cognitive processes: Approaches and applications. Neuropsychologia , 24(1), 151-168. doi: 10.1016/00283932(86)90049-7
Brydges, C. R., Fox, A. M., Reid, C. L., \& Anderson, M. (2014). Predictive validity of the N2 and P3 ERP components to executive functioning in children: A latent-variable analysis. Frontiers in Human Neuroscience, 8, 1-10. doi: 10.3389/ fnhum.2014.00080

Chernyshev, B. V, Lazarev, I. E., Bryzgalov, D. V, \& Novikov, N. A. (2015). Spontaneous attentional performance lapses during the auditory condensation task : An ERP study. Psychology \& Neuroscience, 8(1), 4-18. doi: 10.1037/h0101029

Dalley, J. W., Everitt, B. J., \& Robbins, T. W. (2011). Impulsivity, compulsivity, and top-down cognitive control. Neuron, 69(4), 680-694. doi: 10.1016/j.neuron.2011.01.020

Delorme, A., Mullen, T., Kothe, C., Akalin Acar, Z., BigdelyShamlo, N., Vankov, A., \& Makeig, S. (2011). EEGLAB, SIFT, NFT, BCILAB, and ERICA: New tools for advanced EEG processing. Computational Intelligence and Neuroscience, 1-12. doi: 10.1155/2011/130714

Faber, L. G., Maurits, N. M., \& Lorist, M. M. (2012). Mental fatigue affects visual selective attention. PLOS ONE, 7(10), 1-10. doi: 10.1371/journal.pone.0048073

Fairclough, S. H., \& Venables, L. (2006). Prediction of subjective states from psychophysiology: A multivariate approach. Biological Psychology, 71(1), 100-110. doi: 10.1016/j. biopsycho.2005.03.007

Falkenstein, M., Hoormann, J., \& Hohnsbein, J. (1999). ERP components in Go/Nogo tasks and their relation to inhibition. Acta Psychologica, 101(2-3), 267-291. doi: 10.1016/S00016918(99)00008-6

Folstein, J. R., \& Van Petten, C. (2007). Influence of cognitive control and mismatch on the N2 component of the ERP: A review. Psychophysiology, 45, 152-170. doi: 10.1111/j.14698986.2007.00602.x 
Friedman-Hill, S. R., Robertson, L. C., Desimone, R., \& Ungerleider, L. G. (2003). Posterior parietal cortex and the filtering of distractors. Proceedings of the National Academy of Sciences of the United States of America, 100(7), 4263-4268. doi: 10.1073/pnas.0730772100

Gergelyfi, M., Jacob, B., Olivier, E., \& Zénon, A. (2015). Dissociation between mental fatigue and motivational state during prolonged mental activity. Frontiers in Behavioral Neuroscience, 9, 1-15. doi: 10.3389/fnbeh.2015.00176

Grabner, R. H., Fink, A., Stipacek, A., Neuper, C., \& Neubauer, A. C. (2004). Intelligence and working memory systems: Evidence of neural efficiency in alpha band ERD. Cognitive Brain Research, 20(2), 212-225. doi: 10.1016/j.cogbrainres.2004.02.010

Hart, S. G., \& Stavenland, L. E. (1988). Development of NASATLX (task load index): Results of empirical and theoretical research. Human Mental Workload, 52, 139-183. doi: 10.1016/ S0166-4115(08)62386-9

Hartmann, L., Sallard, E., \& Spierer, L. (2015). Enhancing frontal top-down inhibitory control with Go/NoGo training. Brain Structure and Function, 221, 3835-3842. doi: 10.1007/ s00429-015-1131-7

Hopstaken, J. F., Linden, D. van der, Bakker, A. B., \& Kompier, M. A. J. (2015). A multifaceted investigation of the link between mental fatigue and task disengagement. Psychophysiology, 52(3), 305-315. doi: 10.1111/psyp.12339

Hsu, B.-W., Wang, M.-J. J., Chen, C.-Y., \& Chen, F. (2015). Effective indices for monitoring mental workload while performing multiple tasks. Perceptual and Motor Skills, 121(1), 94-117. doi: 10.2466/22.PMS.121c12x5

Ishii, A., Tanaka, M., \& Watanabe, Y. (2014). Neural mechanisms of mental fatigue. Reviews in the Neurosciences, 25(4), 469-79. doi: 10.1515/revneuro-2014-0028

Jonkman, L. M. (2006). The development of preparation, conflict monitoring and inhibition from early childhood to young adulthood: A Go/Nogo ERP study. Brain Research, 1097(1), 181-193. doi: 10.1016/j.brainres.2006.04.064

Jung, T. P., Makeig, S., Humphries, C., Lee, T. W., McKeown, M. J., Iragui, V., \& Sejnowski, T. J. (2000). Removing electroencephalographic artifacts by blind source separation. Psychophysiology, 37(2), 163-178. doi: 10.1111/14698986.3720163

Kaiser, S., Unger, J., Kiefer, M., Markela, J., Mundt, C., \& Weisbrod, M. (2003). Executive control deficit in depression: Event-related potentials in a Go/Nogo task. Psychiatry Research - Neuroimaging, 122(3), 169-184. doi: 10.1016/ S0925-4927(03)00004-0

Kato, Y., Endo, H., \& Kizuka, T. (2009). Mental fatigue and impaired response processes: Event-related brain potentials in a Go/ NoGo task. International Journal of Psychophysiology, 72(2), 204-211. doi: 10.1016/j.ijpsycho.2008.12.008

Kok, A. (1997). Event-related-potential (ERP) reflections of mental resources: A review and synthesis. Biological Psychology, 45(1-3), 19-56. doi: 10.1016/S0301-0511(96)05221-0

Kok, A. (2001). On the utility of P3 amplitude as a measure of processing capacity. International Journal of Psychophysiology, 30(1-2), 67. doi: 10.1016/S0167-8760(98)90168-4

Lage, G. M., Gallo, L. G., Cassiano, G. J. M., Lobo, I. L. B., Vieira, M. V, Salgado, J. V, ... Malloy-Diniz, L. F. (2011). Correlations between impulsivity and technical performance in handball female athletes. Psychology, 2(7), 721-726. doi: 10.4236/ psych.2011.27110

Lal, S. K. L., \& Craig, A. (2002). Driver fatigue: Electroencephalography and psychological assessment. Psychophysiology, 39(3), 313-321. doi: 10.1017. S0048577201393095

Lange, J. J., Wijers, A. a., Mulder, L. J. M., \& Mulder, G. (1998). Color selection and location selection in ERPs: Differences, similarities and "neural specificity. Biological Psychology, 48, 153-182. doi: 10.1016/S0301-0511(98)00011-8
Lorist, M. M. (2008). Impact of top-down control during mental fatigue. Brain Research, 1232, 113-123. doi: 10.1016/j. brainres.2008.07.053

Lorist, M. M., Klein, M., Nieuwenhuis, S., De Jong, R., Mulder, G., \& Meijman, T. F. (2000). Mental fatigue and task control: Planning and preparation. Psychophysiology, 37(5), 614-625. doi: 10.1111/1469-8986.3750614

Melina, A., Keller, B., \& Coelho, R. W. (2013). Associação entre pressão arterial e estresse percebido em motoristas de ônibus. Journal of the Health Sciences Institute, 31(1), 75-78.

Menon, V., Adleman, N. E., White, C. D., Glover, G. H., \& Reiss, a L. (2001). Error-related brain activation during a Go / NoGo response inhibition task. Human Brain Mapping, 12, 131-143. doi: 10.1002/1097-0193(200103)12:3<131::AIDHBM1010>3.0.CO;2-C

Murata, A., Uetake, A., \& Takasawa, Y. (2005). Evaluation of mental fatigue using feature parameter extracted from event-related potential. International Journal of Industrial Ergonomics, 35(8), 761-770. doi: 10.1016/j.ergon.2004.12.003

Neubauer, A. C., \& Fink, A. (2003). Fluid intelligence and neural efficiency: Effects of task complexity and sex. Personality and Individual Differences, 35(4), 811-827. doi: 10.1016/S01918869(02)00285-4

Neubauer, A. C., \& Fink, A. (2009). Intelligence and neural efficiency: Measures of brain activation versus measures of functional connectivity in the brain. Intelligence, 37(2), 223-229. doi: 10.1016/j.intell.2008.10.008

Pandey, a K., Kamarajan, C., Tang, Y., Chorlian, D. B., Roopesh, B. N., Manz, N., ... Porjesz, B. (2012). Neurocognitive deficits in male alcoholics: An ERP/sLORETA analysis of the $\mathrm{N} 2$ component in an equal probability Go/NoGo task. Biological Psychology, 89(1), 170-82. doi: 10.1016/j. biopsycho.2011.10.009

Park, J., Kim, Y., Chung, H. K., \& Hisanaga, N. (2001). Long working hours and subjective fatigue symptoms. Industrial Health, 39(3), 250-254. doi: 10.2486/indhealth.39.250

Pereira, C. A., Salles, G. C. S., \& Passos, J. P. (2010). As condições de trabalho e sua relação com a saúde dos trabalhadores condutores de transporte. Revista de Pesquisa: Cuidado é Fundamental, 2(1), 904-907. doi : 10.9789/2175-5361.2010.v0i0.\%25p

Phillips, R. O. (2015). A review of definitions of fatigue - And a step towards a whole definition. Transportation Research Part F: Traffic Psychology and Behaviour, 29, 48-56. doi: 10.1016/j.trf.2015.01.003

Polich, J. (2007). Updating P300: An integrative theory of P3a and P3b. Clinical Neurophysiology, 118(10), 2128-2148. doi: 10.1016/j.clinph.2007.04.019

Quirino, G. de S., \& Villemor-Amaral, A. E. de. (2015). Relação entre estresse e agressividade em motoristas profissionais. Revista Psicologia e Saúde, 7(2), 125-132.

Rutiku, R., Martin, M., Bachmann, T., \& Aru, J. (2015). Does the P300 reflect conscious perception or its consequences? Neuroscience, 298, 180-189. doi: 10.1016/j.neuroscience.2015.04.029

Sheppard, L. D., \& Vernon, P. A. (2008). Intelligence and speed of information-processing: A review of 50 years of research. Personality and Individual Differences, 44(3), 535-551. doi: 10.1016/j.paid.2007.09.015

Shomstein, S. (2012). Cognitive functions of the posterior parietal cortex: Top-down and bottom-up attentional control. Frontiers in Integrative Neuroscience, 6, 1-7. doi: 10.3389/ fnint.2012.00038

Smith, J. L., Smith, E. A., Provost, A. L., \& Heathcote, A. (2010). Sequence effects support the conflict theory of N2 and P3 in the Go/NoGo task. International Journal of Psychophysiology, 75(3), 217-226. doi: 10.1016/j.ijpsycho.2009.11.002

Ting, P., Hwang, J., Doong, J., \& Jeng, M. (2008). Driver fatigue and highway driving: A simulator study. Physiology \& Behavior, 94(3), 448-453. doi: 10.1016/j.physbeh.2008.02.015 
van der Linden, D., Frese, M., \& Meijman, T. F. (2003). Mental fatigue and the control of cognitive processes: Effects on perseveration and planning. Acta Psychologica, 113(1), 45-65. doi: 10.1016/S0001-6918(02)00150-6

Wascher, E., Rasch, B., Sänger, J., Hoffmann, S., Schneider, D., Rinkenauer, G., ... Gutberlet, I. (2014). Frontal theta activity reflects distinct aspects of mental fatigue. Biological Psychology, 96, 57-65. doi: 10.1016/j.biopsycho.2013.11.010

Woods, D. L., Wyma, J. M., Yund, E. W., Herron, T. J., \& Reed, B. (2015). Factors influencing the latency of simple reaction time. Frontiers in Human Neuroscience, 9, 131. doi: 10.3389/ fnhum.2015.00131

Wronka, E., Kaiser, J., \& Coenen, A. M. L. (2013). Psychometric intelligence and $\mathrm{P} 3$ of the event-related potentials studied with a 3-stimulus auditory oddball task. Neuroscience Letters, 535(1), 110-115. doi: 10.1016/j.neulet.2012.12.012

Zhao, C., Zhao, M., Liu, J., \& Zheng, C. (2012). Electroencephalogram and electrocardiograph assessment of mental fatigue in a driving simulator. Accident Analysis and Prevention, 45, 83-90. doi: 10.1016/j.aap.2011.11.019 